available evidence is scanty and unclear so this seems a bold assertion. Other authors ${ }^{7,8}$ have been unable to find any dedifferentiating features in any of the blastema constituting cells.

(4) The results reported by Gremigni and Miceli ${ }^{9}$ supporting cell dedifferentiation and transdifferentiation in planarians, though offering new ways to re-evaluate the problem of blastema formation, are not as conclusive as has been suggested. First of all, the sequence proposed: germ cell $\rightarrow$ blastema cell $\rightarrow$ muscle cell, and its corollary of dedifferentiation and metaplasia are not necessarily related as the loss of an haploid complement during spermiogenesis, though one of the first steps from neoblasts to male germ cells, is only a trivial phenomenon regarding cell determination. Unless spermatids or primary spermatocytes are shown to give rise to blastema cells, and then to differentiated cells, we cannot talk properly of dedifferentiation and metaplasia. At the most, this would qualify for dedetermination (or transdetermination) and the sequence proposed should be read instead as predetermined or determined: germ cell $\rightarrow$ blastema cell $\rightarrow$ muscle cell. Second, and more important, the sequence suggested by Gremigni and Miceli, if proved correct, has to be demonstrated for other differentiated cell types since several planarian species, being asexual, lack germ cells. Besides, it is possible, as suggested by Betchaku ${ }^{4}$ and Pederson $^{8}$, that both processes (neoblasts and metaplasia) occur during planarian regeneration. If this were the case, the important issue would be to know the partitioning between both phenomena during planarian regeneration.

$(\overline{5})$ Though most (but not all, witness Hydra) regenerative phenomena in the Animal Kingdom have been proved to occur through cell dedifferentiation and redifferentiation, the present trend to extrapolate this mechanism to all animal groups (including planarians) overlooks the possible relationship between the actual mechanism of regeneration and the complexity of the species. Planarians are one of the most primitive acoelomates, and their tissular complexity is rather low if compared with other epimorphic-regenerating organisms like Amphibia or Insecta. No wonder that dissimilar mechanisms may operate during regeneration in these groups. The planarian neoblast system, based on a unique and multipotential self-renewing stem cell, though appropiate to the low-level complexity of planarians, is clearly

\footnotetext{
Slack, J. M. W. Nature. 286, 760 (1980)

Wolft, E. in Regeneration (cd. Rudnick, D.) 53 (Ronald Pross Co. New York 1962)

Lindh, N. O. Ark.Zool, 10, 497 (1957).

. Betchaku, T. J.exp.Zool. 164, 407 (1967).

5. Sugino, P. H., Okunn, Y. \& Yoshinobu, I. Mem, Osaka \& Kyoiku. Univ. 19,63 (1976).

Hori, I. J. Electron Microsc. 27. 89 (1978)

Peri. K. J. Withelm Roux's Archs 169, 134 (1972)

Gremigni, V. \& Miceli, C. Wilhelm Roux's Archs 188, 107 (1980).

(1980).

11. Slack, J. M. W, J.theor. Biol, 82, 105 (1980)
}

inadequate for organisms with static (decaying) cells and transit cells that require different determined stem cells placed in different body regions. Consequently, the dedifferentiation process, necessary for Amphibia regeneration, is not necessary for organisms like planarians which are in a continuous state of rapid cell turnover ${ }^{10}$. Unless new data on planarian histology and cytology, cell dynamics, and life cycle are obtained, the extrapolation of other mechanisms of regeneration to planarians ${ }^{1,9}$ and the construction of theoretical models of planarian regeneration ${ }^{11}$ will be of little value.

J.M.W. Slack comments: In my article I attempted to show that several independent issues are involved in establishing the source of cells for regeneration, each of which must be approached through a different experiment. As Baguña implies, the simple dichotomy - neoblasts versus metaplasia' - is often not an adequate statement of the problem. I believe that the controversy which has arisen in this field in the past has largely been caused by an imprecision in the question asked.

J.M.W. Slack is in the Imperial Cancer Research Fund Laboratories, Mill Hill, London.

\section{A carbohydrate signal for intracellular transit}

\section{from Mike Geisow}

JUST over a year ago, the function of the oligosaccharide chains added to many newly synthesized proteins was discussed in these columns (Sugars and intracellular recognition, Nature 281,$15 ; 1979)$. It was noted that an early hypothesis - that carbohydrate acted as a marker for secretion - had been superseded by the discovery of the signal peptide. However, it did seem as if carbohydrate on nonsecreted proteins might still act as a signal for intracellular transit from the endoplasmic reticulum to subcellular, membrane-enclosed destinations. Recent work has shown that this is indeed the case for lysosomal enzymes.

Characteristically, lysosomal enzymes are glycoproteins and many have been shown to contain mannose residues phosphorylated at carbon-6 in the hexose rings. Both glycosylation and phosphorylation occur in the endoplasmic reticulum and appear to be directed by the sequence and conformation of the polypeptide substrate; not all proteins receive both modifications. The importance of both the

Mike Geisow is in the National Institute for Medical Research, Mill Hill, London. mannose and the phosphate groups in receptor-mediated processes first became apparent when glycoproteins expressing both determinants were shown to be rapidly and specifically endocytosed by fibroblasts. Next, the uptake of a variety of lysosomal hydrolases was found to be subject to competitive inhibition by mannose phosphate. These observations suggested three rival routes for lysosomal enzymes from their sites of synthesis to primary lysosomes. Two of these, involving secretion followed by re-uptake or cycling as receptor complexes via the surface membrane, appear to be of minor importance: receptor-saturating levels of extracellular mannose-6-phosphate do not deplete lysosomal enzyme levels. Fischer and his colleagues ( $J$. biol.Chem. 255, $9608 ; 1980$ ) now present firm evidence for a major intracellular transit system using $\beta$-hexosaminidase $B$ in fibroblasts.

It was found that 80 per cent of the mannose-6-phosphate receptors were intracellular and faced the interior of endoplasmic reticulum or lysosomes. The highest specific activity of enzyme-binding receptors was present in these cell fractions. Moreover, the intracellular receptors were combined with endogenous enzymes and presented an occupancy gradient which ran steeply downhill from endoplasmic reticulum to lysosomes. The inference is that proteins which receive phosphorylmannose in the endoplasmic reticulum as a result of as yet unknown conformational signals in the polypeptide, are recognized and transferred to primary lysosomes by an essentially intracellular route.

In the report referred to earlier, attention was drawn to the frequent observation of secretary protein proteolysis in endocrine cells treated with inhibitors of protein glycosylation. In such cells, failure of the glycosylation system presumably produces not only nonglycosylated hormones, but also carbohydrate-free acid hydrolases cosynthesized in the endoplasmic reticulum. What happens to the non-glycosylated lysosomal enzymes? In I-cell disease, defective addition of phosphorylated carbohydrate to the enzymes results in their secretion. Co-packaging of acid hydrolases and normal secretory products in the acid environment of a secretion granule may well explain the observed aberrant proteolysis. This argument leads to an interesting thought - is such proteolytic attack on hormones always abnormal? Some hydrolases appear to be naturally present in secretion granules as opposed to lysosomes. These are the conversion enzymes which give rise to products as diverse as insulin, oxytocin and enkephalins from the original precursors. Do such proteases arrive at their destination in the secretory granule because they normally lack a mannose-6phosphate-containing oligosaccharide chain? 